\title{
Consumptive Behavior, Promotional Preferences, And Shopping Patterns Of Hispanic Americans: An Empirical Perspective
}

\author{
Elias G. Rizkallah, La Sierra University, USA
}

Andrew Truong, La Sierra University, USA

\begin{abstract}
Hispanic Americans are becoming a substantial purchasing force in the United States, thus creating as many opportunities as challenges for marketers in many companies. Although the literature is rich with studies of this sub-culture, new information is always welcome due to the extensive changes that this segment of society is experiencing, from population growth, increasing purchasing power and income, to shifting demographics, diversity, and acculturation. This study is designed to enhance understanding about Hispanic Americans via a survey of a random sample of 120 Hispanic American adult individuals in the Inland Empire area of Southern California. The data collected was analyzed and in this paper, the findings are reported and discussed as well as some marketing tactics recommended. At the strategic marketing level, the authors recommend the application of a theoretical marketing framework, one that matches the nature of the Hispanics' market and its dynamics, to develop winning strategies and guidelines for marketers who are interested in this vital market segment.
\end{abstract}

Keywords: Consumptive Behavior, Hispanic Americans, Ethnicity, Promotional Preferences, Shopping Patterns, Media habits.

\section{INTRODUCTION}

¿

$t$ is not surprising to see that the explosive growth of the Hispanic population and their purchasing power in the American marketplace has been surpassed by an intense growth in interest in this market segment. However, until recently, the growing interest in the Hispanic sub-culture in the United States has been challenged by the unwillingness to bend strategies and adjust tactics. Companies today are awakened to the importance of this strategic opportunity and to the adverse effects on their position in the marketplace if they should fail to take advantage of it. In response to this, the last two decades have witnessed a surge in research and learning about Hispanic consumers' demographics, psychographics, diversity, and their consumptive and purchasing behaviors. These new studies have hoped to guide the development of effective strategies and tactics for reaching out to these consumers and seizing this opportunity.

The marketing landscape in the U.S. is changing, and one of the central groups creating that change is the Hispanics. Given their growing impact on the market, it is important to understand who the members of the Hispanic community are. Hispanics are members of a very diverse group in terms of socioeconomic positions, religions, racial classifications, and national origins. They now comprise the U.S.'s largest minority. According to the Census in 2000, 35.3 million people, or about 13 percent of the American population, were Hispanic. In 2004, this number had already increased by 14 percent to 40.4 million (Soto, 2006). There are several important trends that characterize the Hispanic population in the US. (1) It is growing at a faster rate than the rest of the country and any other subculture, (2) the age distribution of this group show them as younger, on average, than the rest of the country, (3) their average income level, although still below the overall average of the U.S., is growing and thus 
giving them even more economic power in the market. According to Hispan Telligence, U.S. Hispanic purchasing power surged to nearly $\$ 700$ billion in 2005 and was projected to pass $\$ 1$ trillion soon thereafter (Soto, 2006). (4) Hispanic Americans are from many countries in Latin America, making them a diverse group, although the majority do come from Mexico. (5) Hispanics tend to acculturate rather than assimilate - they largely preserve their cultural traits while adopting a complementary set of cultural dimensions from the American mainstream. More importantly, they have different levels of acculturation.

Marketers who are interested in Hispanic Americans are faced with several pressing and challenging issues:

- The Hispanic market segment is growing, but can the growth rate be predicted accurately enough to assess the magnitude of this market segment and whether special marketing efforts and budgets are justified, especially under the current and unclear immigration situation and political dynamics between the US and Latin American countries?

- The Hispanic market is diverse, so can this market segment be considered one large segment or does it need to be divided into multiple sub-segments, and are these sub-segments viable? For example, the Hispanic market consists of consumers at different acculturation levels. This may pose a problem and require a differentiated marketing approach to these varying levels of acculturation.

- $\quad$ The Hispanic market is dominated by youth. Hispanic youth, especially those under the age of 18 , constitute one of the largest and most complex demographic groups in the U.S., a group that largely intends to keep a foot in both cultures (Gardyn, 2001). Reaching youth in general is a problem for marketers and reaching today's Hispanic youth can pose some serious challenges.

- Hispanics have their own cultural and behavioral differences that distinguish them from mainstream Americans, thus affecting their purchasing habits, family decision making, brand loyalty, preferred language to communicate in, media habits, etc. The challenge is to determine to which extent these cultural and behavioral differences and their effects are stable over time.

In this study, 120 Hispanic consumers were surveyed in the Inland Empire area of Southern California. The data was collected and analyzed. Findings are reported, discussed, and some recommendations are presented below. The motivation for the study was on the one hand, to learn more about the Hispanic market segment in order to assist practicing marketers in reaching out to this segment with the right products, at the right places and prices, and with the right messages. On the other hand, it is an attempt to bring to the attention of marketers the changing nature of this market segment, and recommend applying a theoretical marketing framework that calls for a continuous monitoring of this segment, and guides the development of winning strategies and tactics.

\section{LITERATURE REVIEW}

Most regions of the United States have experienced a growth in their Hispanic population in the last two decades (Korzenny and Korzenny, 2005). Researchers, academics and practitioners agree that research on the marketplace behavior of Hispanics is an important issue due to the community's size, distinctiveness, significant growth in population and income, and thus purchasing power. However, due to the perpetual growth of the Hispanic community, at least for the foreseeable future, many important aspects of this community need to be studied, including diversity within this major market segment, behavioral patterns attributed to the level of acculturation and age structure, and many cultural and demographic sub-aspects such as language, citizenship status, entry into the U.S., networks, intermarriage, identity, religion, age, education, and income. These characteristics impact the consumptive and purchasing behavior of the Hispanic community, making a detailed understanding of this subculture imperative to the marketers who are interested in this important market segment. It is to be noted that this review is only a glance at some of the work that pertains to the study on hand.

To understand the Hispanic community, one has to approach the task with caution. The assumption that it is one large homogenous community is utterly mistaken. A deeper look into the attributes of this culture is warranted, since it is theorized that the behavior of the Hispanic community can be predicted when one understand its values and characteristics. 
The Hispanic community is one that is very much based on a family oriented culture. To shop with one's family, to spend time with one's family, and to reciprocate opinions from family members and friends is not only normative but a way of life. Studies by Gardyn and Fetto illustrate that 36 percent of all Hispanic families prefer to shop with at least one member of the family and over 25 percent say that kids have a significant impact on their purchasing decisions, such as the brand that they buy. The adhesion of family values seems to have an impact on consumptive and purchasing behavior (Gardyn and Fetto, 2003).

The theoretical model of family communication patterns was addressed in a study by Rafeedie, Godkin, Valentine and Swerdlow. In this study, the researchers found that Hispanics have a more positive relationship and network with family members than their Caucasian counterparts. Through family communication patterns, members of the Hispanic community can use this network as a way to socialize and as a reference. The authors also found that in the absence of the said family communication pattern, one can relate to peers as a reference group (Rafeedie, Godkin, Valentine and Swerdlow, 2006).

In the above study, it was found that in a protective family, the parent or parents will dictate the behavior of their adolescent. Therefore, when marketing efforts are directed at Hispanic teens, marketers should consider promotions that include parental figures for the families with protective family communication style. Conversely, in a pluralistic family communication pattern, parents have less to say regarding purchasing behavior of the adolescents; television and the Internet are considered to be key prompts to purchasing. Further it was concluded that because Hispanic adolescents are raised within protective family communication patterns and have strong links to family and reference groups, family members have a major impact on each other's consumptive and purchasing decisions. In addition, Hispanics from a protective family communication pattern are more concerned with brand names that build their own personal status, and are likely to remain with a brand that provides this benefit (Rafeedie, Godkin, Valentine and Swerdlow, 2006).

Research also suggests that the purchasing decision, the physical behavioral act of purchasing, is directly related to personal influences (e.g., values, attitudes, backgrounds, beliefs, etc.), referent groups, or the media (Kim and Kang, 2001). However, Kim and Kang distinguish between different types of products (i.e., value expressive and utilitarian) that are purchased as a result of the varying impact of these influences. Understandably, it is unlikely that that referent groups will have a large impact on the purchase of utilitarian products. However, when purchasing value expressive products, like high priced products similar to televisions, appliances, or motor vehicles, the opinions of the referent network are more likely to dictate purchasing behavior (Kim and Kang, 2001)

Furthermore, Hispanics value the positive relationships and networks developed with family members and friends more than their Caucasian counterparts. "The significance ascribed to values such as familialism (the importance of relatives as referents and as providers of emotional support) and to social scripts such as "simpatia" (the preference for positive interpersonal interactions), are characteristics shared by most Hispanics" (Marin \& Marin, 1991). These two principles are seen in the power and respect given to authority figures in Hispanic society (Rafeedie et al, 2006).

The Spanish language within the Hispanic community is the tie that binds and the one that supersedes national boundaries. However, it would be naïve to uniformly lump all of the foregoing nationals into one category. For example, the amount of Spanish used in the home and whether it is the predominant form of communication will dictate subcategories of Hispanics. Arjona proposes that language is an indicator that can determine which subcategory one belongs to (Arjona et al, 1998). For instance, if Spanish is seldom spoken at home, one can be labeled as being assimilated. Conversely, if Spanish is the only language spoken at home, a Hispanic person can be categorized as being isolated. Additionally, if both Spanish and English are interchangeably spoken at home, then one is considered to be acculturated. Now, given these subcategories of Hispanics, one can see that marketing to the entire community requires more than a one size fits all approach. What does language have to do with cultural identity? The idea is simple. Language perpetuates thinking, thinking drives certain attitudes and values, and ultimately values and attitudes dictate behavior (Korzenny and Korzenny, 2005). Korzenny's work argues that connotations from different languages encapsulate different images. For a Spanish speaker, an ad depicting a Mexican national calling his hometown and speaking Spanish would paint a more vivid, relatable picture than if the advertisement was to be ran in English (Korzenny and Korzenny, 2005). So, does this mean that every 
advertisement, spoken or in print, should be in Spanish? This is far from so. When asked if they prefer Spanish advertisements to the English ones, approximately 53\% disagreed. Yet, when it relates to broadcast media as a whole, the Hispanic community tends to favor Spanish as the medium (Kim and Kang, 2001). Accordingly, the language spoken in commercials or utilized in print is not an approach that one can use as a blanket when addressing the Hispanic community as a whole. Most Spanish speakers speak Spanish to preserve a sense of cultural identity and yet they see the need to learn English as a part of life here in the United States. It is no accident that the majority of the Hispanic community is acculturated or bicultural (Soto, 2006); in that, to be acculturated or bicultural, one retains his culture while taking on a secondary culture. Other studies have attempted to understand the implications of an acculturated or bicultural Hispanic community, to find that acculturation impacts consumer behavior in a variety of ways including: (a) language preferences in advertising, (b) affinity towards race/ethnicity of models used in advertisements, (c) advertisement recall, (d) attitude towards advertisements, (e) preference for certain types of retail outlets, (f) consumption patterns, and (g) importance attached to certain product attributes (Ueltschy and Krampf, 1997; Kara and Kara, 1996).

Furthermore, the next generation of Hispanic youth intends to keep a foot in both cultures. If marketers want to reach these young consumers, they will have to do the same (Gardyn, 2001). Hispanic youth are the largest ethnic youth population, accounting for $17 \%$ of all those under age 18. Hispanics under the age of 18 constitute one of the largest and most complex demographics in the nation. Unlike their parents and grandparents, who felt compelled to, at least publicly, melt into the American pot; this generation of consumers - representing 35\% of all Hispanics - wants the best of both worlds. As a result, the Spanish-only media channels that keep their parents entertained and informed are not always enough, and marketing to them in their own language no longer means simply translating general market strategies into Spanish. More culturally relevant media vehicles and advertising messages, better media planning, and bigger budgets are a must for any marketer trying to build brand loyalty and relationships with tomorrow's mainstream consumers, as those consumers are and will increasingly be of Hispanic origin. So while reaching the adult Hispanic population is probably easy - an ad on Spanish-language media such as Univision or Telemundo usually does the trick - reaching today's Hispanic youth can cause some serious headaches (Gardyn, 2001). To this effect, major TV networks are giving options in the form of more flexible advertising policies. So, for an advertiser to be effective with today's Hispanic youth market, he needs to be everywhere they are, and with a message that is relevant to them, both in Spanish and in English. More than that, marketers will have to set aside bigger budgets to develop ads that speak to Hispanic youth and build strong relationships with them.

In general, the media plays a positive role in influencing purchasing behavior. However, that influence varies with media type and ethnicity. For example, when compared with other ethnicities in the U.S., the media, particularly television and radio, has a slightly greater effect on Hispanics (Kim and Kang, 2001). Also, research is inconclusive about which language is more effective in television and radio ads - research results do not demonstrate that Hispanics prefer Spanish to English. Another study investigated the effects of language (i.e., English vs. Spanish), brand loyalty, preference for shopping at neighborhood stores and the propensity to purchase prestigious brands, in advertising to Hispanics (Korgaonkar, Karson and Lund, 2001). Findings indicated that the Hispanic community prefers print advertising in Spanish. Also, the study found brand loyalty to be a significant factor in purchasing behavior, more specifically loyalty to prestigious or national brands (Korgaonkar, Karson and Lund, 2001; Gardyn and Fetto, 2003). Conclusions also have been drawn indicating that Hispanic consumer preferences and shopping patterns are not any different than that of their Caucasian counterparts. Hispanics, like other Americans, prefer low prices, convenient locations, and a wide range of merchandise (Soto, 2006).

Many magazine advertisers employ numerous Hispanic models to enhance advertising effectiveness in reaching the Hispanic target market. Studies showed that minority group members tend to have more favorable attitudes towards advertising and those with more favorable attitudes toward advertising in general find specific advertisements (with favorable models included) more acceptable, informative, and enjoyable (Peterson, 2007). Further positive attitudes toward advertising can lead to favorable attitudes toward the brand and, ultimately to purchase. Members of minority groups are more likely to be market mavens ---they diffuse information to others, are aware of new products earlier, provide information to other consumers across product categories, engage in general market information seeking, and exhibit general market interest and attentiveness (Peterson, 2007). 
Further evidence suggests that foreign-born Hispanics prefer employees of companies and stores to speak Spanish, products related to or relevant to Hispanics, signage in Spanish, packaging and labels in Spanish, and a wide assortment of payment options (Soto, 2006). In this study, when a comparison was made between foreignborn Hispanics and US-born Hispanics, the former group shows a greater affinity toward these shopping preferences. One can deduce here that it will be more beneficial to study and examine shopping and purchasing behavior and preferences of Hispanics within subgroups -- US-born and foreign-born; subgroups of different acculturation levels - isolated, acculturated (bicultural) or assimilated; etc.

Finally, recognizing the importance of multicultural marketing, many companies have responded to the lure of this fast growing ethnic market, and dedicated business units to focus on research, site planning and merchandise distribution directed at the Hispanic community. Advertisers targeting Hispanics have developed advertisements, emphasizing their unique cultural values, based on findings indicating that Hispanics tend to gravitate toward products they recognize and relate to. For example, if popular trademarks sold well in their countries of origin, typically these goods continue to be purchased by them in the United States. So, for retailers, this information raises questions such as: Are the stores carrying specialty foods or the appropriate mix of merchandise that appeal to Hispanic consumer? Are there bi-lingual employees available to answer questions and to help make their shopping experiences more pleasurable? Is there Spanish-language signage directing these consumers to various products and services? Many companies are seeking to make these changes in response to the preferences of the Hispanic consumer. For example, Wal-Mart has worked with two major companies, McDonald's and Subway, when putting fast-food restaurants in its stores. But now, in a move that reflects the changing demographics of its shoppers - and the country --- the retail giant is making room for three new chains in its stores, two of which (Pollo Campero and Taco Maker) have a Hispanic flair (Businesswire.com, September 24, 2007).

Summing up, this review can be summarized in a well articulated statement by Tim Kregor, president of Nielsen Consumer Panel Services: "It is critical for retailers and marketers to understand the wide range of factors driving Hispanic consumers' shopping behavior....By understanding what Hispanic consumers are buying, where they're buying it, how they're buying it and why, retailers and marketers can adapt product offerings and promotions to ultimately better satisfy this rapidly growing and diverse consumer segment (Business wire.com September 24, 2007).

\section{METHODOLOGY}

Data for this study was collected from 120 Hispanic adult (over 18 years old) immigrants residing in the Greater Inland Empire area in California. The respondents were randomly intercepted in Hispanic markets located in the Inland Empire. These areas were specifically selected due to the high concentration of Hispanic immigrants in them. A total of 289 shoppers were approached, but only 120 were willing to complete the questionnaire to the extent that can be used in the analysis.

The respondents were told that the primary purpose of the study was to obtain information about their purchasing habits, product preferences, favorite promotions, major influences on their purchasing decisions, store preferences, and finally, some demographic information about them.

The survey utilized a self-administered questionnaire consisting of structured questions. The questionnaire first addressed some general questions about the respondents' frequency of shopping for groceries and nongroceries, who shops in the family, and the stores they most frequently visited and why. Then the respondents were asked more specific questions regarding their preferences for promotional tools (e.g., price discounts, deals, coupons, rebates, premiums, etc), and sources of information (word-of-mouth, newspapers, magazines, TV, Internet, direct mail, radio, posters, etc.). These questions were then followed by a set of attitudinal statements (using 5-point Likert Scale ranging from strongly agree to strongly disagree) pertaining to their brand loyalty, purchasing influences, preferences, and their attitude towards advertisement and brands that incorporate touches of Hispanic heritage, and more. One section of the questionnaire was dedicated to media preferences and TV viewing habits. Finally, the questionnaire ended with a few questions about the respondent's demographics, including country of birth and how long they have been in the United States if born in another country. 
To ensure the validity of the study, the respondents were offered a choice between an English or Spanish questionnaire. Seventy-one respondents completed an English language questionnaire and forty-nine completed the same questionnaire in Spanish.

\section{RESULTS}

The demographics of the respondents included eighty-seven (73.1\%) males, and thirty-two (26.9\%) females. The age distribution ranged between 18 and over 75 years with approximately 45.7 percent of the respondents reporting an age between 26 to 45 years old. About half of the respondents (49.6\%) were born in the United States, and 50.4 percent reported having been born outside the United States. Those who were born outside the U.S. immigrated at different ages that varied from one year old to sixty seven years old - a majority of them (73\%) came to the U.S. at 23 years of age or younger. About 39 percent of the respondents claimed the U.S. as their country of origin, about 61 percent claimed a country outside the U.S. as their country of origin. Of those 61 percent, about 65 percent claimed Mexico as their country of origin and the other 35 percent claimed other countries (Peru, Chile, Cuba, Colombia, El Salvador, Guatemala, Nicaragua, Puerto Rico, Honduras, Argentina, and Brazil). As far as how long they had been in the United States, 32.8 percent of respondents had been living in the U.S. for more than 20 years, 14.3 percent had been living in the U.S. for 11-20 years, 8.4 percent for 4-10 years, and only 4.2 percent had been living in the U.S. for 1-3 years.

A slight majority of the respondents (53.8\%) reported Spanish as their primary language, and 45.4 percent noted English as their primary language. As far as the size of their household, 31.6 percent reported having 1-2 individuals, 41.2 percent reported 3-4 individuals, and 27.2 percent reported five or more individuals in their household. Fifty-three percent of the sample reported that they owned their house, 35 percent rented their house, and 12.0 percent lived with someone. As for marital status, 48.7 percent were married, 31.9 percent were singles, and 4.2 percent were widowed, 5 percent divorced, and 10 percent were in a partner relationship.

Sixty percent reported having children living at home, of those 26.2 percent reported having children under twelve year old, 24.6 percent reported having children twelve to eighteen years old, and 22.1 percent reported having children over eighteen years of age at home. Regarding the educational level of the respondents, 6.6 percent reported having some or completing elementary school, 18.7 percent having some or completing high school, 47.5 percent reported having some college or technical school, 14.4 percent reported completing 4 year college, and 12.7 percent completing graduate or postgraduate studies. As for occupation of the respondents, 54.8 percent reported a white collar job, 22.6 percent reported a blue collar job, 6.7 percent were homemakers, and 12.2 percent reported other jobs (part-time student worker, retired, etc.). The distribution of income was as follows: 26.1 percent made under $\$ 20,000,27$ percent made $\$ 20,000-40,000,15.7$ percent made $\$ 40,001$ to $\$ 60,000,18.3$ percent made $\$ 60,001$ to $\$ 80,000$, and 13.1 percent made more than $\$ 80,000$ per household. It is to be noted that the levels of education and income of the sample are skewed upward, perhaps due to the fact that within the area of the sample population, there are four universities and two colleges.

The survey reported that 5.1 percent of respondents indicated that they shopped for food or groceries daily, 19.5 percent reported twice a week, 36.4 percent reported weekly, and 24.6 percent reported biweekly. When cross tabulated with age, the younger the respondents were, the more often they shopped -- 36.4 percent of 26-35 year olds reported that they shopped twice a week while, of the older group (46-55 years of age), only 13.6 percent shopped twice a week $(\mathrm{p}=.004)$. A plausible explanation for this is that the older respondents have families, so they stock up more and thus shop less frequently than younger individuals who tend to eat out and not much at home.

As for the frequency of shopping for nonfood items, the respondents reported less frequent trips than when they shopped for food. The majority (63.3\%) shopped every two weeks or more. When asked whether someone accompanies them when going shopping, 42.9 percent of the respondents reported that they go shopping for groceries alone, 26.1 percent reported that they shop for groceries with a spouse, 12.6 percent with children, 6.7 percent with other family members, and 7.6 percent with friends. According to the results, families with children at home usually bring them along when going shopping for groceries. And if they do not have children, they tend to go with their friends $(\mathrm{p}=.001)$. Findings confirm that the majority of Hispanics are more likely to go shopping accompanied by someone. 
When surveyed about who would influence their product selection when buying groceries, 36.8 percent reported spouse, 14.5 percent reported children, 5.1 percent reported friends, and 43.6 percent reported other stimuli. It is interesting to note that friends have a greater influence on purchasing decisions of respondents with lower levels of education than on those with higher levels of education; while respondents with higher education are more influenced by their spouse and children $(\mathrm{p}=.005)$.

When asked who shops for groceries, 57.1 percent reported that the mother of the family shops for groceries, 9.2 percent reported that the father shops for groceries, 33.6 percent reported other family members. The larger the household size, the more likely that the mother is the shopper for groceries $(\mathrm{p}=.004)$.

According to the survey the most visited super markets, in order of the most to least visited, were Stater Brothers, Food 4 Less, Vons, Costco, Albertson, and Ralph's. Supermarkets characterized as Hispanic (i.e., El Tapatio and Cardenas) were mentioned towards the bottom of the list. Stater Brothers and Food 4 Less supermarkets can be considered mainstream but with a heavy emphasis on catering to Hispanics. The rest of the supermarkets listed are considered mainstream although they have lately been trying to capture a share of the Hispanic market segment.

In the case of shopping for non-grocery items, the most visited stores were as follows starting from the most visited to the least: Wal-Mart, Target, Macy's, JC Penney, Ross, and Kohl's. When asked about the reasons for shopping in their most frequented store, 64.2 percent checked because it is close to home (convenience), 60 percent checked price, 45.8 percent quality of products, 36.9 percent mentioned product variety, and 15 percent customer service.

A majority (72.5\%) of the respondents shop in Hispanic stores. Primary language reported has a significant effect on shopping at a Hispanic store -- 89.9 percent of respondents who reported Spanish as their primary language said they shop in Hispanic markets versus 57.4 percent of those who reported English as their primary language $(\mathrm{p}=.001)$. A panel study from ACNielsen on grocery purchasing habits of Hispanic consumers in the Los Angeles area showed a definite preference for smaller mom-and-pop stores among Spanish-speaking-only or Spanish-speaking-preferred household. Conversely, Hispanic families that are bilingual or prefer to speak English patronize the larger stores that offer better prices. Ken Greenberg, Director of Marketing for ACNielsen Homescan Consumer Panel, attributed the differences to two factors. The first, he said Hispanics new to an area may feel more comfortable with a small neighborhood grocery store that offers ethnic food products they are familiar with. But as they become more acculturated, Hispanics' preferences shift toward the big stores that feature better prices and more services; and the other factor is availability of transportation to get to the larger stores (Howell, 1999).

When asked about shopping in Hispanic stores, respondents mentioned visiting the following stores starting with the most to the least visited: Cardenas, El Tapatio, Ranch Market, Superior, Maxi Food, and some mentioned Libario and Fiesta Food. Reasons for shopping in Hispanic stores were checked as follows: for lower prices (38.3\%), for quality (13.5\%), to find Hispanic products (51.7\%), because it is easy to communicate (10.7\%), and for the fresh produce (19.2\%). When lower price preferences were cross tabulated with household income level, those with lower income favored shopping in Hispanic stores for the lower prices $(\mathrm{p}=.01)$. Primary language reported by respondents has a significant relation with shopping in Hispanic markets to find Hispanic products $(\mathrm{p}=.008)$. Of those who reported Spanish as their primary language, 64.1 percent said they shop in Hispanic markets for Hispanic products versus 37.0 percent of those who reported English as their primary language. Also, when cross tabulated with educational level, respondents with lower educational levels expressed a higher interest in shopping in Hispanic markets because of ease of communication than those with higher levels of education $(\mathrm{p}=.000)$.

According to the results, Hispanics prefer to shop in an actual store, followed by on-line as a second preference and then via catalogues. Age played a significant role in preference for on-line shopping. The younger the respondents, the more likely to shop on line $(\mathrm{p}=.000)$.

When asked what influenced their purchasing the most among these factors---price, quality, brand name, package, store, and familiarity with the brand, the respondents ranked these factors consecutively from most important to the least important as such: price, quality, familiarity with the brand, brand name, store, and package; 
with only a slight difference between brand name and the store preference. Respondents who have been in the United States a longer period of time exhibited a tendency to place more emphasis on quality, price and brand name $(\mathrm{p}=.000)$. Their preferences approached those who are born in the U.S.

Regarding preference and usage of sales promotional techniques, respondents' preferences were as follows: Discounts (46.7\%), coupons (34.4\%), and lastly premiums (e.g., a gift with the purchase). However, when asked about the most used, findings were as follows: Coupons (43.4\%), discounts (41.0\%) and premiums maintained third place in usage as well (4.1\%).

Regarding the purchase of frozen and pre-prepared food, frequency of purchase was used as a measure and the results were as follows: 40.2 percent of respondents reported buying frozen food weekly or bi-weekly and 35.3 percent reported buying every month or every other month, and 22.1 percent never bought frozen food. For preprepared food, 34.4 percent buy weekly or bi-weekly, 36.9 percent buy monthly or every other month, and 26.2 percent never bought pre-prepared food. There was a significant difference $(p=.010)$ between frequency of buying pre-prepared food and household size. As the family size got larger, the tendency to buy pre-prepared food was less frequent, however when the family size was smaller, respondents showed a tendency to buy pre-prepared food more frequently. Also, respondents who reported English as their primary language tended to buy pre-prepared food more frequently than those who reported Spanish as their primary language $(\mathrm{p}=.007)$, showing a trend toward acculturation if language is considered a part of that process.

When asked about the most effective promotion for each product category, the results were as follows: Discounts and deals were checked as most effective for food and household supplies, discounts and deals for clothing; discounts and mail-in rebates for electronics and household appliances. In food purchasing, the longer the respondents have been living in the U.S., the higher the tendency to consider coupons as an effective promotional technique. The same applies for discounts, and deals $(\mathrm{p}=.000)$. However, in purchasing household supplies, the longer they have been in the U.S., the higher the tendency to consider discounts and deals as most effective ( $\mathrm{p}=$ $.000)$. Also, there is a significant difference in preference for promotional tools and household size $(\mathrm{p}=.001)$. The larger the household size the more likely to prefer discounts, mail-in-rebate, and then deals as the most effective promotional tools.

Upon examining the responses to the attitudinal statements, the results revealed that 50.8 percent of respondents will buy the brands that their parents used to buy and about 58.2 percent said they buy products familiar to them from their country of origin. At the same time, 59.0 percent strongly agree or agree that they like to try new products including new ethnic products. A slight majority of respondents expressed interest in buying brand name products. When asked about their attitude towards products and services that use the Spanish language, appeal to Hispanic cultural values, and/or include touches of Hispanic heritage in their commercials, about 38-40 percent showed a positive attitude towards these products and services. About 27 percent of respondents watch TV commercials attentively, 40 percent said TV commercials are informative and 40.2 percent don't mind on-line advertisements. A majority of respondents $(60.6 \%)$ were willing to visit a store if they were informed that a sale was going on, and 87.7 percent agreed to the statement "I look for a bargain when I go shopping". It is important to note that 81.9 percent of the respondents reported that they will repeat purchase a brand if they like it - this gives a sense of confirmation to what the literature cites about Hispanics as brand loyal. Also, 74.6 percent attested that they are more likely to purchase a brand if they are more informed about it. When cross-tabulated with demographic variables of the respondents, only primary language and the statement "I like to try new brand" was significant ( $\mathrm{p}=$ .003 ). Those who reported English as their primary language were more likely to try new brands than those who reported Spanish as their primary language.

According to the survey results, the most important sources of information were, in order, word of mouth, and then TV, magazines, newspaper, direct mail and Internet. However, when asked about which advertising vehicles influences them the most, respondents marked TV (50.8\%), followed by word-of-mouth (41.8\%), Internet (22.1\%), Radio (19.7\%), Newspaper (18.0\%), Magazine (17.2\%), direct mail (12.3\%), Flyer/Brochure (13.1\%), and Posters $(3.3 \%)$. 
A majority of the respondents view mainstream TV channels available through cable or satellite broadcasting companies; a few respondents mention certain Spanish language channels. Forty-seven percent of the respondents spend 2-3 hours watching TV on a daily basis, 32.0 percent watch one hour or less, and about 9.8 percent watch for four or more hours. When asked about newspapers they read, only 54.9 percent of the sample answered that question, with the majority of them reading their local newspaper or the closest major city newspaper. A very small percentage read national newspapers or Spanish language newspapers.

Eighty-four percent of the respondents have Internet access. Sixty-one percent use the Internet to pay bills, Forty-one percent to buy on-line, 76.2 percent to search for information, 69.7 percent for E-mail, and 15.6 percent for other activities. When asked if they would consider making a purchase on-line, 66.4 percent said yes. Also, the results showed that the younger the respondents, the more likely they were to have Internet access and the more likely they were to use it $(\mathrm{p}=.001)$. Also, the longer they have been living in the U.S., the higher the tendency to use the Internet to search for information $(\mathrm{p}=.001)$. According to Hispanic News (www.hispanic.bz), there are 12.4 million Hispanic online users in the U.S. (Korgaonkar, Silverblatt, and Becerra, 2004), and the number of online users is far higher today.

\section{CONCLUSIONS AND RECOMMENDATIONS}

The results of this survey largely confirmed the findings in the literature except in a few instances. This can be attributed to the fact that the selected sample had more educated, more affluent, and younger respondents than the general Hispanic population. However, the study offers important implications for marketing managers who are interested in the Hispanic market segment in the U.S. in general and for those interested in the Hispanic community in the Inland Empire area of Southern California in particular. It is the interest of the authors in this paper to present the recommendations within a theoretical step-wise model of marketing planning for companies (manufacturers, service providers, or intermediaries) interested in this vital segment of the market. The following are the steps of the model, followed by some tactical marketing recommendations based on the survey:

- Is the current Hispanic sub-population in the U.S. market of importance to the organization and its strategic position in the market place? And if not, would it be of strategic importance within the foreseeable future? At this point, the viability of this strategic opportunity for the company is determined.

- If the answer is yes in either case, then this issue needs to be brought to the attention of top management and addressed at that level in order to gain the necessary support and commitment. It will require patience and passion to succeed in capturing this segment. Most importantly, interest should be followed by actions.

- The next step is to determine the relationship between the company's offerings and the Hispanic market segment along several dimensions - compatibility, ability to satisfy Hispanics' unmet needs and wants, appeal to specific aspirations, characteristics, and lifestyles, etc. In this exercise, the marketer will be challenged by many questions about Hispanic household in the U.S. - Who are they? Are they one large homogeneous segment or many diverse sub-segments? And if so, what segmentation bases should be used? ---country of origin, level of acculturation, age, etc. There is strong evidence in the literature that encourages and recommends dealing with the Hispanic market segment as diverse sub-segments.

- Evaluate the sub-segments and the adaptation requirements to assess the potential profitability of each subsegment and determine the company's target sub-segment(s) within its scope of business.

- Study each selected sub-segment closely to optimize resource allocation, determine brand positioning, and develop appropriate marketing tactics which offer the product/service in the right form, with the right attributes, at the right price, in the right place, and with the right communication and preferred promotional techniques.

The Hispanic segment within the US is a wide and diverse group with varying backgrounds and demographics, and ever-changing characteristics. It is also an increasing proportion of the population. For these reasons, it is important to continue to monitor these groups in order to obtain a better understanding of their consumer spending patterns, what shape these may take in the future, and the impact they will exert on your organization and your competitors in the market place. The assumption that all Hispanics are one large segment is at the very least impractical. One must approach this segment with an open mind and caution. You must always research your target segment with the intention of finding out specific results that guide marketing decision-making. 
The following are some recommendation extrapolated from the survey and discussion.

On the marketing tactics front, the findings can provide some insights to develop the marketing program. Hispanics of the Inland Empire are more educated and younger than the community on average. Whether they are newly arrived to the U.S. or are born in the U.S., they are being acculturated through their interaction with the American culture while simultaneously keeping a warm connection to their heritage. They appreciate their families and strong family relationships, their ethnic food, and many traditions, customs, and lifestyles from their own culture. Many factors such as language, length of stay in the U.S., and education are affecting their level of acculturation. Their level of acculturation and income is in turn determining the extent to which they are conforming to mainstream patterns of purchasing and consumption.

Regarding the product component of the marketing program, it is important to note that Hispanics are like everybody else. They are interested in trying new products and even other ethnic products. They are also interested in brand names and, if they can afford them, are likely to develop brand loyalty, provided that the brand satisfies their needs and wants. They are not easily lured by packaging, but are definitely into quality. Therefore, for a marketer to create a relationship between their brands and the Hispanic target market, they need to devote themselves to genuine adaptation efforts. In some cases, these may not require any changes to the product, but on the other extreme, a company may need to create new offerings that are consistent with Hispanic preferences.

The challenge for a marketer is how to do this while maintaining reasonable prices or generous promotions, since value and a bargain are important to the Hispanic shopper. Price was at the top of the list of the most influential factors in the purchasing decision. Therefore, integrated efforts along the value chain, to reduce cost and maintain a reasonable level of quality, in order to offer lower prices would be helpful until this segment of the market catches up on the affluence dimension.

According to the results of the survey, Hispanics prefer to shop and buy their products in big supermarkets. This is especially true if they perceive these stores to be offering some ethnic Hispanic products and reasonable prices. The results showed that this was the case for Stater Brothers and Food 4 Less supermarkets. Respondents were also attracted to the low prices at discount stores like Wal-Mart and Target, and department stores like J.C. Penney. Nevertheless, Hispanics (and most likely those of low income, who speak only Spanish, and are less educated) will still go to Hispanic stores for specific ethnic ingredients and the low prices these stores offer. Companies interested in this market segment should seek to have a presence in those stores and supermarkets. Furthermore, it is important for neighborhood stores to be receptive and welcoming to Hispanics by having some employees who speak the language, and maximizing the product types that appeal to Hispanics within their area. If Hispanics are brand loyal, as mentioned above, it will be beneficial to the stores to stock or continue to stock those brands favored by Hispanics to reinforce brand loyalty and build store loyalty, since it is very likely that Hispanics will continue to frequent a store because they have had success in obtaining their favorite brands from that store (Berkowitz, Bao, and Allaway, 2005). It is important to note that although Hispanics prefer to shop in an actual store, there is a tendency to favor on-line shopping as an alternative, especially when targeting young Hispanics.

Companies seeking to maximize the benefits of their promotional budget should consider the costs and benefits of addressing each segment. Familiarity with the brand ranked as the third most influential factor preceded by price and quality. Family exerts a great deal of influence on purchasing decisions of Hispanic individuals, especially the educated since, according to the survey, they are more influenced by family members' opinions than Hispanics with lower educational levels. For those with lower levels of education, friends had a greater influence on their purchasing decisions. However, both groups highly appreciate word-of-mouth as a source of information. To capitalize on this, marketers should create a buzz in the market place, starting with Hispanic youth and reaching out to all family members through communication. Discounts and coupons are the most preferred promotional tools.

Language encapsulates culture and tradition, and is essential in creating a sense of belongingness. Direct translation will not work, as Spanish is more than just a language; it is the carrier of cultural identity. One must always check translations to see if the intended message is carried out correctly. Radio is best for local markets. The internet is a growing medium for Hispanics regardless of backgrounds and demographics. Putting a human face to a promotion increases Hispanic consumers' level of trust and belief in the program. Promotions that draw on 
culturally relevant, popular marketing assets such as musical acts, sports, pastimes, or entertainment add to Hispanic consumer interest. It is important to focus on reaching the target market with culturally sound promotions. Keep it simple and believable, hold information requests to a minimum, and be mindful of lifestyle issues, empower Latinos through interactive games of chance, and inject a human element through bilingual brand ambassadors. Have a passion for the Hispanic market and allow your customers to participate in your decisions and implementation. Employ Hispanics on the marketing team -- Hispanics who work in Hispanic marketing have it easier because they usually need fewer things explained to them. For example, they can read, understand, and develop creative advertisements and cultural cues that are in Spanish, and many idiosyncrasies of the market are second nature to them.

Overall, the results, even with the study's limitations in terms of sample size and being confined to a small geographic market, contribute to the literature by providing greater understanding of Hispanic purchasing and consumptive behavior. Further research is a worthwhile investment for marketers interested in building a lifetime relationship with one of the fastest-growing groups in the United States.

\section{AUTHOR INFORMATION}

Elias G. Rizkallah is a professor of marketing at School of Business, La Sierra University, Riverside, California. Educational background -- Ph.D. in marketing from Northwestern University and MBA in marketing and quantitative analysis from New York University. Research interests: International marketing, strategic planning, and consumer behavior.

Andrew Truong is assistant professor of management for the School of Business with La Sierra University. His academic vitae includes a graduate degree in operations management and finance from California Polytechnic University in Pomona and a doctorate degree from the University of California in Los Angeles. His current research interests possess multi-discipline assessment tools to investigate organizational effectiveness, which intersects the areas of finance, logistics, management and marketing.

\section{REFERENCES}

1. Arjona, Luis D., Shah, Rajesh, Tinivelli, Alejandro, and Weiss, Adam (1998), "Marketing to the Hispanic Consumer" The McKinsey Quarterly, 3, pp. 107-114.

2. Berkowitz, David, Bao, Yeqing, and Allaway, Arthur W. (2005), "Hispanic Consumers, Store Loyalty and Brand Preference" Journal of Targeting, Measurement and Analysis for Marketing, (September), 14, 1, pp. 9-24.

3. Business wire.com September 24, 2007)

4. Gardyn, Rebecca (2001), "Habla English?” American Demographics (April); 23, 4, pp. 54-57.

5. Gardyn, Rebecca, and Fetto, John (2003), "Race, Ethnicity and the Way We Shop: Understanding the Different Attitudes and Behaviors of Asian, Black, Hispanic and White Consumers." American Demographics (February), pp. 30-33.

6. Howell, Debbie (1999), "Spanish-speaking Hispanics Prefer Neighborhood Shops." Discount Store News, (October 4), p. 4.

7. Kara, Ali, and Kara, Natasha R. (1996), "Ethnicity and Consumer Choice: A Study of Hispanic Decision Processes Across Different Acculturation Levels." Journal of Applied Business Research, (Spring), 12, 2, pp. 22-35.

8. Kim, Youn-Kyung, and Kang, Jikyeong, (2001), "The Effects of Ethnicity and Product on Purchase Decision Making.", Journal of Advertising Research, (March-April), pp. 39-48.

9. Korgaonkar, Pradeep, Karson, Eric, and Lund, Daulatram (2001), "Direct Marketing: A Comparison of Hispanic and Non-Hispanic Perspectives." International Journal of Advertising, 20, pp. 25-47.

10. Korgaonkar, Pradeep, Silverblatt, Ronnie, and Becerra, Enrique (2004), "Hispanics and Patronage Preferences for Shopping From the Internet." JCMC.indiana.edu (April), 9, 3, pp.1-18.

11. Korzenny, Felipe, and Korzenny, Betty Ann (2005), Hispanic Marketing: A Cultural Perspective, Elsevier Inc.; Burlington, MA. 
12. Marin, G., and Marin B.V. (1991), Research with Hispanic Populations. Sage: Newbury Park, California.

13. Peterson, Robin T. (2007), "Consumer Magazine Advertisement Portrayal of Models by Race in the US: An Assessment.” Journal of Marketing Communications (September), 13, 3, pp. 199-211.

14. Rafeedie, Christina, Godkin, Lynn, Valentine, Sean, and Swerdlow, Robert A. (2006), "The Development of a Model Specifying the Differences in Hispanic and White Adolescents' Consumer Behavior." International Journal of Management, (September), 23, 3 Part 2, pp. 597-605.

15. Russell, Jeremy (2002), “Targeting Hispanic Consumers", The National Provisioner, (November), pp. 4453.

16. Soto, Terry J. (2006), Marketing to Hispanics: A Strategic Approach to Assessing and Planning Your Initiative, Kaplan Publishing; Chicago, Illinois.

17. Ueltschy, L.C., and Krampf, R.F. (1997), “ The Influence of Acculturation on Advertising Effectiveness to the Hispanic Market" Journal of Applied Behavioral Research, 13, 2, pp.87-101. 